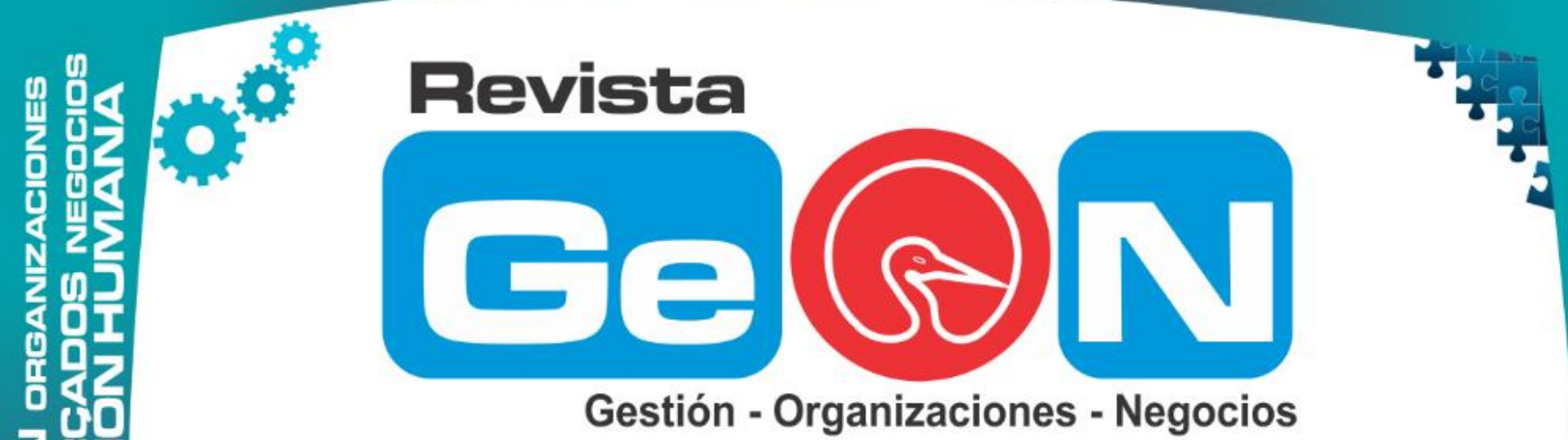

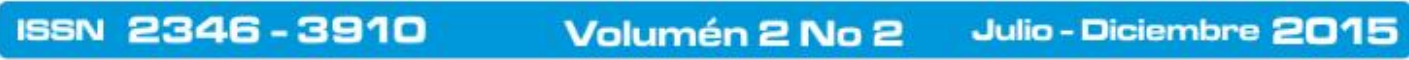

Revista Electrónica de la Facultad de Ciencias Económicas de la Universidad de los Llanos

1. 3 II

440

VILLAVICENCIO - COLOMBIA

$>2$

64

2

2 平品

70

ব)

展

o

ii III

0 U

ใด

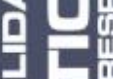

居的

임

Iiin in

照

只

造

iil 2

if in

4 吕

2

400

付 iI
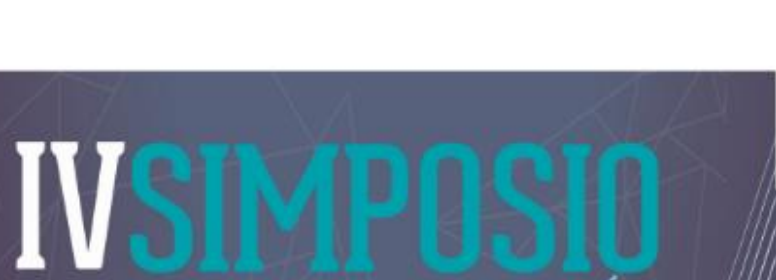

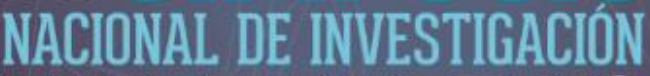

EN MARKETING
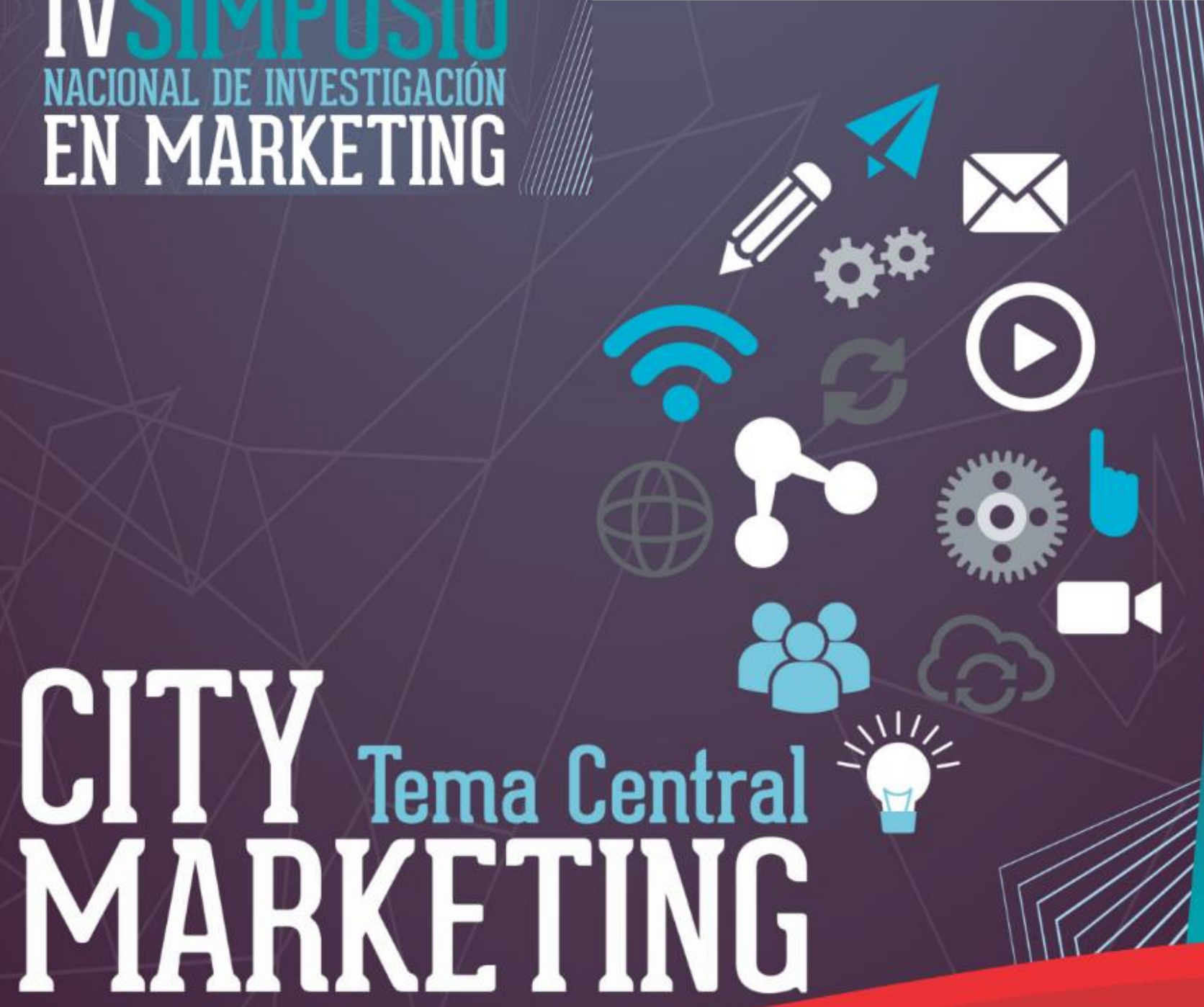

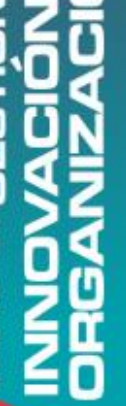
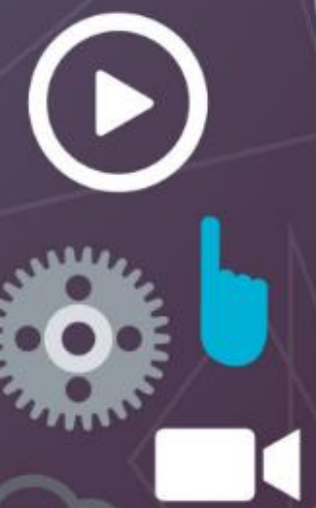


\section{Propuesta de medición de la efectividad de la publicidad en la credibilidad de las celebridades - Germán Quintero}

Germán Augusto Quintero Duarte, MBA énfasis en Mercadotecnia (C) Pdh en Marketing, gaquinte@gmail.com

\section{Resumen}

La publicidad es un mecanismo que influye en la adquisición de un producto o servicio para finalizar una transacción. En la actualidad los artistas reconocidos en el ámbito nacional e internacional influyen en la generación de credibilidad en la negociación entre el oferente y el consumidor. Estas celebridades muestran su imagen a través de productos de publicidad lo que garantiza el éxito de dicha negociación. En el artículo se presenta una propuesta para la medición de la efectividad de esta publicidad en el consumidor final basada en la escala de Ohanian (1990), considerando el caso de Shakira y James Rodríguez como personalidades que manejan su imagen a través de la publicidad y que influyen en la credibilidad de los productos o servicios que promocionan.

\section{Palabras clave}

Credibilidad; publicidad; actitud; consumidor; efectividad.

\footnotetext{
Abstract

Publicity is a mechanism that influences the purchase of a product or service to complete a transaction. Currently renowned artists in national and international fields influence the generation of credibility in the negotiation between the suppliers and the consumer. These celebrities show their image through advertising products which guarantees the success of negotiation. The article presents a proposal for the measurement of the effectiveness of this advertising in the consumer based on the scale of
}

Ohanian (1990), considering the case of Shakira and James Rodríguez as personalities who manage its image through publicity and affecting the credibility of the products or services that they promote.

\section{Keywords}

Credibility; advertising; attitude; consumer; effectiveness.

\section{Introducción}

La publicidad es un elemento de gran importancia que permite a las organizaciones llegar de manera directa a los consumidores. La actitud del consumidor hacia el anuncio a través de radio, televisión e inclusive internet, influye en que su intención de compra varíe, de acuerdo a la marca que promociona una celebridad. La percepción que se genera a través de los anuncios influye en la credibilidad de la celebridad para la adquisición del producto. Precisamente, en este documento se presenta un modelo de medición para la influencia de la credibilidad de dos personalidades colombianas en la negociación entre una empresa y un consumidor. Concretamente se estudia el caso de Shakira y James Rodríguez, como personalidades que influyen en la decisión de compra final. La escala de Ohanian (1990) facilita el establecimiento de un modelo de validación de escala que permita analizar la relación entre la credibilidad medida a partir de la honradez, experiencia percibida y atractivo, con la actitud del consumidor hacia la publicidad, la marca y su intención de compra.

Este artículo hace parte de una investigación que se está desarrollando en la Universidad de Santander, UDES, relacionada con la propuesta de la validación de escala de la credibilidad de los artistas y su influencia en la intención de compra de los consumidores. 


\section{Referente teórico}

La credibilidad medida a través de la experiencia percibida, honradez y atractivo

El concepto de credibilidad es objeto de análisis en el área del marketing, concretamente en el ámbito de la publicidad, debido a la importancia de mostrar en el mercado anuncios que proyecten credibilidad al receptor (Goldsmith, et al., 2000).

Según Ohanian (1990) la credibilidad se refiere a la capacidad del mensaje de proyectar experiencia o "expertismo" (expertise), es decir, la capacidad de mostrar que el emisor sabe, comprende y es experto en lo que habla, siendo capaz de proyectar confianza. Así, la experiencia percibida se deriva del conocimiento que muestra la fuente emisora sobre el objeto del mensaje, mientras que la confianza se basa principalmente en la honradez que proyecta el emisor (McGinnies y Ward, 1980).

La credibilidad se compone de las variables: experiencia, honradez (Ohanian, 1990) y el atractivo (Goldsmith et al., 2000). De hecho, Goldsmith et al. (2000) indica que el atractivo hace parte de la credibilidad citando a McGuire en sus investigaciones del año 1958. Otros autores han considerado esta variable como parte del constructo "credibilidad" y la han incluido en sus trabajos de investigación (Baker y Churchill, 1977; Caballero y Solomón, 1984; DeSarbo y Harshman, 1985). Ohanian (1990) decidió incluir el atractivo como dimensión de la credibilidad en la escala que creó en 1990, sugiriendo que el atractivo físico del comunicador tiene un efecto positivo sobre la opinión del emisor del mensaje. Según Goldsmith et al. (2000), la dimensión de la credibilidad es aplicable cuando se refiere a la belleza de una persona, pero no parece tener efecto sobre el belleza de otro tipo de entes, como en el caso de cualquier objeto que pueda describirse como bello dentro de una corporación. Es así la escala de Ohanian (1990) aplicable al atractivo físico de hombres o mujeres como comunicadores del anuncio.
Goldsmith et al. (2000) y Spry et al. (2009) en sus investigaciones muestran como este tipo de escalas son efectivas para el análisis de los mensajes publicitarios en los que el comunicador es una celebridad, incluyendo para dichos estudios las tres dimensiones mencionadas: experiencia percibida, honradez y atractivo.

En relación con ello, ya desde la década de los cincuenta, autores como Hovland et al. (1953) señalaron que los consumidores son más propicios a ser persuadidos cuando perciben que la fuente proyecta credibilidad. Así, ha sido del interés de los investigadores en publicidad relacionar la credibilidad con la actitud hacia la publicidad, actitud hacia la empresa, producto o marca que se publicita en el anuncio, y la intención de compra (Biel y Bridgwater, 1990; Ohanian et al., 1990; Goldsmith et al., 2000; Lafferty et al., 2002).

De otro lado, Ajzen y Fishbein (2005) indican que existen dos tipos de actitudes hacia los objetivos físicos y generales, $\mathrm{y}$ hacia el objeto $\mathrm{u}$ objetivo, conocidas porque se orientan hacia el comportamiento del ser humano. Esto influye de manera directa en que las personas sigan sus creencias, actitudes e intenciones, lo cual es muy importante en su visión hacia la publicidad y sobre todo a la influencia de una personalidad tras un anuncio.

\section{Influencia de la credibilidad en la decisión de compra del consumidor}

La actitud hacia la publicidad es un término definido como una predisposición aprendida a responder de forma consistente, ya sea favorable o desfavorablemente, a la publicidad en general (Lutz, 1985). Este concepto se refiere a la actitud general de los consumidores hacia la publicidad, y no a la actitud de un determinado consumidor o grupo de consumidores hacia un anuncio determinado o a un tipo de medio (Burns, 2003). En este sentido, el trabajo de Biel y Bridgwater (1990) demostró que la actitud hacia la publicidad consiste en dos dimensiones relacionadas con las creencias de los consumidores: creencias económicas y creencias 
sociales. No obstante, son varios los trabajos que se refieren a la actitud hacia un anuncio determinado, basados en las premisas previas de la actitud hacia la publicidad en general (Zanot, 1984; Zang, 2000; Spry et al., 2009), donde se observa como derivado de otro conjunto de variables, como pueden ser: el medio en el que se publicita, el momento, la forma, el diseño, los sonidos, los comentarios, incluyéndose también como variable relevante la persona que comunica el mensaje, todo ello puede generar un tipo de actitud de los receptores hacia dicha publicidad (Mittal, 1994).

Diversos estudios evidencian que la credibilidad se presenta como una fuente fundamental para entender la actitud de los consumidores hacia la publicidad en general, y sobre la efectividad de un anuncio en particular (Lutz et al., 1985), de hecho, la credibilidad influencia la actitud hacia el anuncio (Biel y Bridgwater, 1990; Ohanian et al., 1990; Lafferty et al., 2002) influyendo en la decisión de compra. Además, se ha establecido que hay una fuerte relación entre una actitud positiva hacia la marca y la intención de adquisición de la misma (p. e. DeSarbo y Harshman, 1985; Lafferty et al., 2002), de esta forma, cuando se produce una actitud positiva hacia una marca en concreto, el consumidor suele tener la intención de comprarla.

\section{Modelos de medición}

Dentro de los modelos de medición se abordan el de Ohanian (1990) y el de Choi y Rifon (2012). Ohanian (1990) centra su investigación en la medición de la efectividad de la publicidad a través del atractivo, de la credibilidad y la experiencia del consumidor. Dicha experiencia se basa en un modelo estadístico medido a través de las Ecuaciones Estructurales, donde se han considerado tres constructos: atractivo, confiabilidad y experiencia, como se observa en la Figura 1:

La investigación fue realizada a 237 estudiantes de 40 universidades y considerando cuatro personalidades exitosas en ese momento en Estados Unidos. Al realizar el análisis de ecuaciones estructurales se detectó que efectivamente dichos constructos eran significativos y apropiados para hacer la medición de la publicidad. Los constructos fueron medidos en una escala Likert entre los extremos bajo sinónimos, como se observa en la Figura 1, así por ejemplo: atractivo, no-atractivo; hermoso, feo; elegante, sencillo; etc.

Source-Credibility Scale
Attractiveness
Attractive-Unattractive
Classy-Not Classy
Beautiful-Ugly
Elegant-Plain
Sexy-Not Sexy
Trustworthiness
Dependable-Undependable
Honest-Dishonest
Reliable-Unreliable
Sincere-Insincere
Trustworthy-Untrustworthy
Expertise
Expert-Not an expert
Eualified-Unowienced-Inexperienced

Figura 1. Dimensiones del modelo de Ohanian (Ohanian, 1990). 
De otro lado, Choi y Rifon (2012) realizan su investigación introduciendo una nueva dimensión para comprender los efectos de las celebridades a través de la publicidad, considerando la percepción del consumidor en las características de la personalidad de la celebridad basadas en el auto concepto que este tiene de las celebridades. Esto influye de manera directa en la decisión de compra y por tanto en la transacción.

\section{Medición de la efectividad de la publicidad}

\section{Metodología}

El objetivo de este artículo es proponer un modelo que a partir de la medición de la actitud hacia la publicidad, permita en una segunda etapa validar la escala de Ohanian (1990), para medir la experiencia percibida, la honradez y el atractivo en las celebridades en Colombia.

Se propone la realización de una encuesta online para ser aplicada a estudiantes de las 30 Universidades asociadas a la Asociación de Programas de Mercadeo - ASPROMER establecidas en Colombia. Este instrumento se basa en la Escala de Ohanian (1990) y medidos en una escala de Likert de 1 a 7 , siendo 1: estoy totalmente en desacuerdo, y siendo 7: estoy totalmente de acuerdo.

\section{Modelo propuesto}

Tras el análisis de la metodología que aplicó Ohanian (1990) en la construcción y validación de una escala para medir la influencia de las celebridades percibida a través de la confianza, el atractivo y la experiencia, se presentan los diferentes ítems que se medirán en la segunda fase del proyecto:

Los ítems que están resaltados son los que han sido propuestos por Ohanian (1990) y validados en su modelo, los subrayados son propuestos por Choi y Rifon (2012), y los demás por el autor basado en la revisión de la literatura.

\begin{tabular}{|c|c|c|c|}
\hline & \multicolumn{3}{|l|}{ Constructo } \\
\hline & Atractivo & Confianza & Experiencia \\
\hline 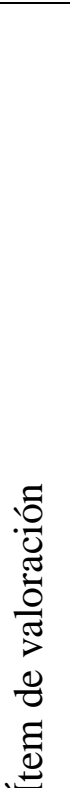 & $\begin{array}{l}\text { Atractivo } \\
\text { Clase } \\
\text { social } \\
\text { Belleza } \\
\text { Elegancia } \\
\text { Sexy } \\
\text { Familiar } \\
\text { Sofisticado } \\
\text { Glamoroso } \\
\text { Encantador }\end{array}$ & $\begin{array}{l}\text { Confiable } \\
\text { Honesto } \\
\text { Fiable } \\
\text { Sincero } \\
\text { Inteligente } \\
\text { Veraz } \\
\text { Ético } \\
\text { Informado } \\
\text { Amable }\end{array}$ & $\begin{array}{l}\text { Experto } \\
\text { Experimentado } \\
\text { Calificado } \\
\text { Cualificado } \\
\text { Conocedor } \\
\text { Partícipe } \\
\text { eventos } \\
\text { Reconocido } \\
\text { Trayectoria } \\
\text { Imagen }\end{array}$ \\
\hline
\end{tabular}

Tabla 1. Constructos del modelo de medición de la efectividad de la publicidad

Fuente: elaboración propia.

\section{Resultados esperados}

En la aplicación del instrumento se espera comprobar que la imagen de las celebridades influye de manera directa en la decisión de compra del consumidor, por la confianza que este le inspira y la experiencia que muestra en determinado campo de acción.

La publicidad mediada por una celebridad es un medio que influye directamente en el consumidor, puesto que al promocionar una marca o un servicio interviene en la decisión de compra del consumidor y por ende en el incremento de sus ventas.

El reconocimiento de las celebridades implica que el consumidor adquiera un producto $\mathrm{o}$ servicio, debido a la credibilidad que se les adjudica por sus carreras profesionales, motivados por su forma de actuar, así como la percepción para el consumidor sobre su imagen.

Uno de los principales aspectos que busca un consumidor para adquirir un producto o servicio 
es la confianza que emana el personaje, producto de su experiencia, honestidad y los valores que muestra tener según sus acciones o actividades que se ven reflejadas en la imagen que pregonan.

\section{Conclusiones}

Es un hecho que la publicidad influye en el consumidor para la adquisición de un producto o servicio. Si a esto se le añade que un artista reconocido en los medios de comunicación, sea quien promociona una marca, la consecuencia directa es que se incrementen las ventas, momento ventajoso para las organizaciones.

Los artistas reconocidos influyen de manera directa en la adquisición de un producto o servicio que estos promueven. Lo anterior respaldado por la credibilidad que se han ganado a lo largo de su carrera y por los hechos que han protagonizado y que por tanto los ha convertido en personajes populares.

La confianza es uno de los principales atributos que requiere un anuncio y propiamente el anuncio que es respaldado por un artista reconocido en los medios de comunicación. Esa confianza que se genera porque el artista muestra ser confiable, honesto, entre otros valores, implica que el consumidor se sienta identificado con lo que promueve y así decida adquirir un producto o servicio que es promovido por el artista.

Otro factor de la publicidad que incide en la decisión de compra del consumidor, mediada por la influencia de un artista, es la experiencia que este demuestra en el conocimiento de un producto o servicio que se presenta a través de la publicidad.

La percepción de los consumidores referente a los comerciales que presentan los artistas en los medios de comunicación influye en la adquisición del producto o servicio que este promociona.

\section{Referencias}

Ajzen, I., \& Fishbein, M. (2005). 5. "The influence of attitudes on behavior". In The handbook of attitudes (pp. 173-222).

Baker, M. J.; Churchill, A. (1977): "The Impact of Physically Attractive Models on Advertising Evaluations". Journal of Marketing Research, 14.

Biel, A. L.; Bridgwater, C. A. (1990). "Journal of Advertising Research", 30, 3. Attributes of likable television commercials.

Caballero, M.; Solomón, P. (1984): "Effects of Model Attractiveness on Sales Response". Journal of Advertising, 13, 1.

Choi, S. M., \& Rifon, N. J. (2012). "It is a match: The impact of congruence between celebrity image and consumer ideal self on endorsement effectiveness". Psychology \& Marketing, 29(9), 639-650.

DeSarbo, W. S.; Harshman, R. A. (1985): "Celebrity-Brand Congruence Analysis. Current Issues and Research in Advertising". MI: Division of Research, University of Michigan, 17-52.

Goldsmith, R. E.; Lafferty, B. A.; Newell, S. J. (2000): "The Impact of Corporate Credibility and Celebrity on Consumer Reaction to Advertisements and Brands". Journal of Advertising, 29, 3.

Hovland, C.; Kelley, H. (1953): "Communication and Persuasion". Yale University Press: New Haven, Connecticut.

Lafferty, B. A.; Goldsmith, R. E.; Newell, S. J. (2002): "The Dual Credibility Model: The influence of Corporate and endorser credibility on attitudes and purchase intentions". Journal of Marketing Theory and Practice, 10, 3.

Lutz, R. J. (1985): "Affective and cognitive antecedents of attitude toward the ad: A conceptual framework". In L. F. Alwitt \& A. A. Mitchell (Eds.), Psychological processes and 
advertising effects theory, research, and application. Hillsdale, NJ: Lawrence Erlbaum Associates.

McGurie, W. J. (1958): "Attitudes and Attitude Change. Handbook of Social Psychology". 2. New York: Random House, 233-346.

Mittal, B. (1994): "Public assessment of TV advertising: Faint praise and harsh criticism". Journal of Advertising Research, 34, 1.

Ohanian, R. (1990): "Construction and Validation of a Scale to Measure Celebrity Endorsers' Perceived Expertise, Trustworthiness, and Attractiveness. Journal of Advertising, 19, 3.
Spry, A.; Pappu, R.; Cornwell, B. T. (2009): "Celebrity endorsement, brand credibility and brand equity". European Journal of Marketing, 45,6 .

Zanot, E. (1984): "Public attitudes towards advertising: The American experience". International Journal of Advertising, 3, 1, 3-15.

Zhang, P. (2000): "The effect of animation on information seeking performance on the World Wide Web: Securing attention or interfering with primary tasks". Journal of Association for Information 